\title{
Coevolution of machines and tapes
}

\author{
Takashi Ikegami * and Takashi Hashimoto ** \\ Institute of Physics, College of Arts and Sciences, University of Tokyo, \\ Komaba 3-8-1,Meguro-ku, Tokyo 153, Japan
}

\begin{abstract}
A problem of self-referential paradox and self-reproduction is discussed in a network model of machines and tapes. A tape consists of a bit string, encoding function of a machine. Tapes are replicated when it is attached by an adequate machine. Generally, a tape is replicated but it may be different from the original one. In this paper, external noise evolves diversity in a system. New reaction pathway induced by external noise will be reproduced deterministically by an emerging autocatalytic network. Hence it will remain stable after external noise is turned off. Low external noise develops a minimal self-replicative loop. When external noise is elevated, a more complex network evolves, where a core structure emerges. Tapes in a core network can be bifurcated into either a RNAlike or a DNA-like tape with respect to its usage in an autocatalytic loop.
\end{abstract}

\section{Introduction}

Origin of life is often attributed to the emergence of self-reproductive properties. People believe that both proteins and RNAs are necessary entities for self-reproduction. Taking RNAs as software, we need hardware to implement the software; i.e., proteins. In the same way, taking protein as hardware, we need some software to drive it; i.e., RNAs.

John von Neumann first proposed an automaton model for the self-reproduction problem [1]. In his abstract modeling, a fundamental problem is clear. To copy something, we first have to observe it. However, observation in some cases disturbs the object.

In addition to the problem of observation and copying, the self-referential problem known as Richard's problem occurs for self-copying. A self-reproducing automaton should interfere with itself for replication of itself. This generates a self-referential paradox [2]. To avoid the difficulty, von Neumann separates a machine from its description tape as well as proteins and RNA/DNAs. In Neumann's model, he defines a tape as a pattern of stationary states as a pattern of active states. If no external disturbance is possible, replication scenario is perfect. However, when other machines come to interact or if external noise causes error actions, replication becomes unstable.

\footnotetext{
^ E-mail address : ikeg@sacral.c.u-tokyo.ac.jp

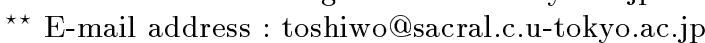


How to encode a machine function on a tape and how to make it stable under noisy environment is our main concern in this paper.

We propose the additional problem of self-replication, as is also stressed by Neumann himself. That is a problem of how to evolve more complex machines from simple self-replicating machines. Random updating of automaton states brings mutations into Neumann's automaton model. If mutations occur in a machine itself, a machine may change its function. But such mutations should not be copied onto the next generation due to Central Dogma. If mutations occur in a tape, it will be copied onto the next generation. Especially neutral mutations will be accumulated in future innovations of machine functions.

In this paper, we will study the evolution of machines and description tapes influenced by random external noise $[3,4]$. While a machine reads a tape, both probabilistic and deterministic errors are assumed to occur. The probabilistic error is caused by external noise and is called a passive error. On the other hand, the deterministic error is caused by machine action and is named an active error.

In low external noise regime, perfect replicating network composed of one or two machines(s) is evolved. In high noise regime, a complex autocatalytic network sustaining deterministic mutation evolves. Self-replication not as an individual but as a network now becomes important. In the other autocatalytic models $[5,6]$, reproduction is discussed only by machines or by tapes. However, the important fact is that no machine can reproduce itself without the coding tapes and vice versa. Because of this restriction, autocatalytic loops of machines only or of tape only will be suppressed. By considering both machines and tapes, we will discuss the dynamics of coding of machines in the present model.

\section{Modeling}

Our system consists of two different objects, tapes and machines.

A tape has a bit string of a circular form. A machine consists of 3 different parts, a head, a tail and a transition table. Each head and tail is expressed by a 4 bit string, whose pattern will be compared with binary patterns of tapes. A transition table consists of 4 mappings; $\left\{\left\{\left(\sigma^{m}, \sigma^{t}\right) \rightarrow\left(\sigma^{\prime m}, \sigma^{\prime t}\right)\right\}\right.$, where $\sigma^{m}$ and $\sigma^{t}$ represents a current binary state of machine and tape. A tape and machine state will change to $\left(\sigma^{\prime m}, \sigma^{\prime t}\right)$, respectively depending on a current state of machine and tape.

Introducing an ensemble of tapes and machines, we carry out the machinetape reaction process as follows:

(1) Interaction of machines and tapes

Machine $\mathbf{M}_{\mathbf{i}}$ reads tape $\mathbf{T}_{\mathbf{j}}$ iff tape $\mathbf{T}_{\mathbf{j}}$ has a head $\mathbf{h}_{\mathbf{i}}$ and tail $\mathbf{t}_{\mathbf{i}}$ in a different site of the pattern. The site from $\mathbf{h}_{\mathbf{i}}$ to $\mathbf{t}_{\mathbf{i}}$ will be called the reading frame.

$$
\mathrm{M}_{\mathrm{i}}+\mathrm{T}_{\mathrm{j}} \rightarrow \mathrm{M}_{\mathrm{k}}+\mathrm{T}_{\mathrm{l}}
$$

Then machine $\mathbf{M}_{\mathbf{i}}$ reads a tape $\mathbf{T}_{\mathbf{j}}$ and rewrites from the site $\mathbf{h}_{\mathbf{i}}$ to $\mathbf{t}_{\mathbf{i}}$ according to the transition tables. A half population of machine starts to read a 
tape with the internal state 1 and the other half does with the state 0 . As the result, it generates a new set of machine $\mathbf{M}_{\mathbf{k}}$ and tape $\mathbf{T}_{\mathbf{l}}$ per each interaction.

During the read/write process, both probabilistic and deterministic errors are assumed to occur. The probabilistic error is caused by external noise and is called a passive error. On the other hand, the deterministic error is caused by machine action, and is named an active error. We call it error since it does not replicate a tape but actively rewrites it. A rewritten tape can be taken as a mis-copy of the original tape. The rate of passive error is measured by the bit flip rate per bit. The active error is measured by the rewriting rate per a length of reading frame when a machine reads a tape. Namely, it is given by,

$$
\mu_{A}=\frac{w}{L}
$$

where a symbol $w$ denotes the number of rewritten bits and $L$ denotes a length of reading frame.

(2) Translation of tapes:

Not only bits of a reading frame, but every bit of tape is repeatedly picked up to construct a new machine from a first site of the reading frame. If a length of a tape is not enough, the same bit is used for coding several different part of a new machine. In the present model, we use a fixed length of 7-bit tapes with 16-bit machines. A first 8 bits are mapped onto head and tail parts in order. The next 8 bits are mapped onto a transition table. In order to cover 16 bits by 7 bits, several bits are multiply used. This complicated mapping from bits of tape onto machine function is assumed to reflect the nonlinear mapping from one-dimensional DNA to 3-dimensional protein folding structure.

(3) Population dynamics:

We presume two abstract spaces of the size $N$ for tapes and machines. A total $m$ machines and $t$ tapes distribute over the respective space. By iterating the following procedures, we simulate the machine/tape reacting system.

1. Compute concentration of tapes $\left(f_{m}\right)$ and machines $\left(f_{t}\right)$ by dividing the population number of each object by the capacity size $N$.

$$
f_{m}^{i}=\frac{m^{i}}{N}, f_{t}^{j}=\frac{t^{j}}{N}
$$

2. Make $f_{i j} N$ numbers of new machines and tapes from the reaction of machine $i$ and tape $j$. The frequency of reaction $f_{i j}$ is given by,

$$
f_{i j}=\frac{c_{i j} f_{i}^{m} f_{j}^{t}}{\sum_{k, l} f_{k}^{m} f_{l}^{t}} .
$$

The coefficient $c_{i j}$ takes a positive constant $c_{m}\left(=c_{t}\right)$, if a machine $i$ can read tape $j$. Otherwise it takes zero value.

3. Remove $d_{m} \%$ of old machines and $d_{t} \%$ of old tapes. 
4. Put the new machines and tapes back in each space. Hence the population of machine $i$ and tape $j$ of the next generation becomes,

$$
\begin{aligned}
m_{i}^{\prime} & =\left(1-d_{m}\right) m_{i}+\sum_{k+j \rightarrow i} f_{k j} N, \\
t_{j}^{\prime} & =\left(1-d_{t}\right) t_{j}+\sum_{k+i \rightarrow j} f_{k i} N .
\end{aligned}
$$

It should be noted here that each machine has its unique description tape but the inverse is not true. Generally each tape encodes several machines depending on which site the translation starts in.

5. Taking an integer part of the above population, we obtain the actual population of the next generation. The machine or tape whose frequency is lower than $\epsilon$ is removed from the population. Not to go beyond the total population size $\mathrm{N}$, the coefficient should satisfy the relation $c_{m / t} \leq d_{m / t}$.

(4)Effect of external noise:

Out of $f_{i j} N$ new tapes, a number of tapes as well as machines, are erroneously generated by external noise. It is assumed that the rate of error depends on the reading frame. Namely, the rate of error replication by external noise is given by,

$$
w=1-\left(1-\mu_{p}\right)^{L},
$$

where the symbol $L$ is the length of reading frame. We use Monte Carlo methods to get the mutant objects. At most $w f_{i j} N$ mutant populations are obtained by randomly flipping the bit within a reading frame.

(5)Reading tapes:

Each tape has a source where an attached machine starts to search for the head and tail pattern. Starting from the site, patterns are searched for in the clockwise direction of a circular tape. When a head pattern is found, a tail pattern starts to be searched in the clockwise direction. The site of source can be updated randomly when the tape is exploited and newly generated. Note that every translational invariant tape has the same source.

\section{Destabilization of a minimal self-replicating loop}

About 10 randomly selected machines with 2 or 3 tapes are prepared as the initial configuration. A machine without description tape is unstable and smoothly removed from the system. Hence an initial configuration which does not include any description tapes of the initial machines will die out if there is no mutation process. External noise may produce description tapes by mistake.

Even without external noise, a machine can acquire its description tape by the other machines as a normal product. To sustain the tape, we have to have the description tape of the machine which generates the tape of the first machine. In order to make it stable, a successive reproducing process should form a closed loop; each machine on the loop reproduces a machine for the next position. 
We will see how the replicating networks evolve by changing the amount of external noise. Examples of temporal evolution of population of machines and tapes are shown below(Fig.1).

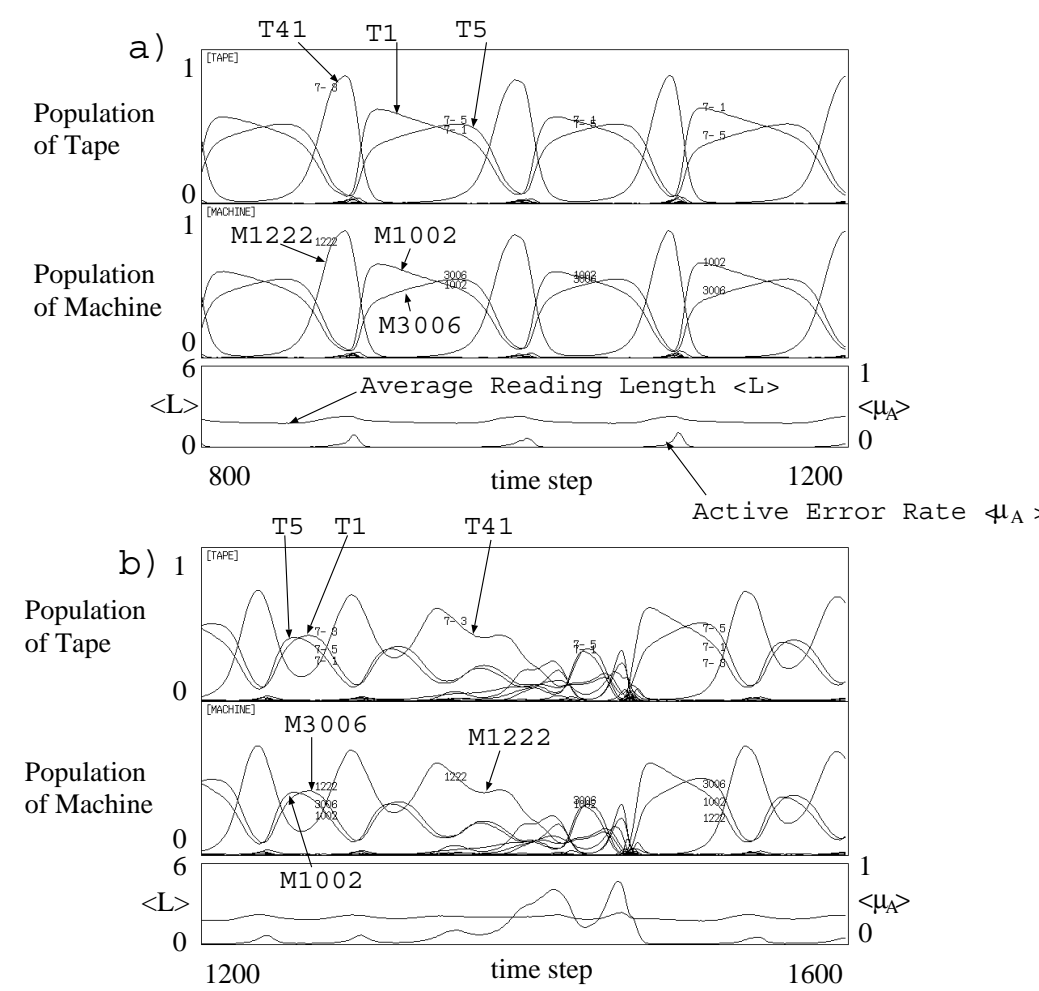

Fig. 1. Temporal evolution of population of machines(the top column)and tapes(the middle column). The bottom column displays temporal evolution of averaged active error rates and the averaged length of the reading frame. The parameters of population dynamics are $c_{m / t}=d_{m / t}=0.6$ in the influence of, a) lower external noise $(=0.04)$ and $\mathrm{b})$ higher external noise $(=0.055)$. Both start from the same initial states.

By introducing a lower amount of external noise in a system, we see a minimal autocatalytic loop evolve. In this example, a machine $\mathbf{M}_{\mathbf{1 0 0 2}}$ reads a tape $\mathbf{T}_{\mathbf{1}}$ to replicate the same tape and its own machine. The number attached to tapes and machines are hexadecimal number converted from its binary representation. Many initial configurations reach this minimal autocatalytic system for a lower noise regime.

A system with the minimal autocatalytic loop is said to be metastable since it remains stable after turning off external noise. However the minimal loop 
is destabilized by increasing external noise. Translation under external noise generates many machines, most of which rewrites existing tapes. Increasing of parasite machines destabilizes the original self-replicating loop. In Fig-1-a), a parasitic machine $\mathbf{M}_{\mathbf{1 2 2 2}}$ invades the network with its description tape $\mathbf{T}_{\mathbf{4 1}}$.

A greater variety of machines and tapes induces unstable oscillation in Fig.1b). An original self-replicating pair becomes unstable if too many parasitic machines attach to it. Population of each machine and tape show unstable oscillation in time. An oscillation of large amplitude is caused by the original self-replicating loop. Other oscillations are caused by parasitic machines and tapes.

This temporal oscillation spontaneously crashes by exhausting the self-replicating loop. The system restarts by having the minimal self-replicating loop, otherwise it is exploited.

It should be noted that a non-zero active error rate begins to oscillate in time in Fig.1-b). A low rate of active errors means that the network has more individual self-replication; each tape is self-replicated without error. But a high active error suggests that many machines read tapes and producing different machines with different tapes from the original ones. Namely, failure of selfreplication is reflected in the amplitude of active errors.

The minimal loop shows zero active error as being depicted in Fig.1-a). Intermittent bursts of active error rates are caused by the parasite machine $\mathbf{M}_{\mathbf{1 2 2 2}}$ and the tape $\mathbf{T}_{\mathbf{4 1}}$.

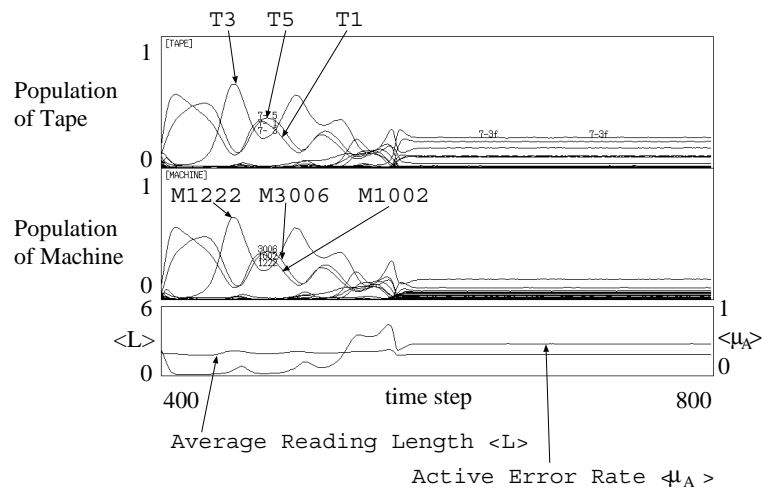

Fig. 2. A spontaneous transition into a core network of a fixed point state. It displays temporal evolution of population of machines(the top column) and tapes (the middle column), and averaged active error rates and the averaged length of the reading frame(the bottom column). The rate of external noise is set at 0.07 . The parameters of population dynamics are $c_{m / t}=d_{m / t}=0.6$. 


\section{Emergence of Core network}

In the region of high external noise $\left(\mu_{p} \geq 0.05\right)$, a stable structure seems to evolve. Unstable oscillation in population amplitude as we see in Fig.1-b) is spontaneously stabilized around time step 600 in Fig.2. At the same time, the active error rate is sustained at the high level.

If we turn off external noise after time step 600, the numbers of machines and tapes remain stable. But if the noise is removed before time step 600, it will back to a minimal self-replicating loop. We call a network which acquired an implicit stable structure a core network.

Fig. 3 shows temporal evolution of machines and tapes after turning off external noise. The amplitude of total number of machines and tapes become less rugged by compared with that before a transition. Generally, machines disappears from the system when turning off the noise. Then a true core network is left in the system.

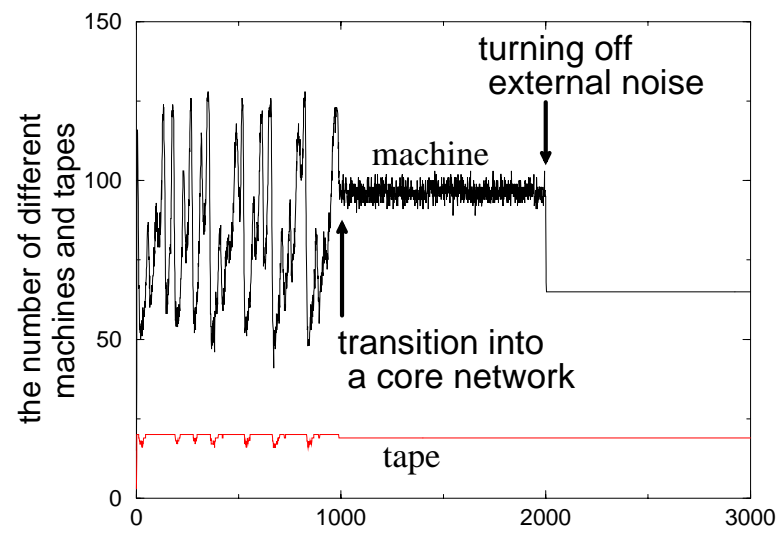

Fig. 3. Temporal evolution of distinct numbers of machines and tapes. External noise $(=0.08)$ is turned off at time step 2000 after the emergence of a core network at time step 1100. The parameters of population dynamics are $c_{m / t}=d_{m / t}=0.6$.

A core network is not necessarily a fixed point state. It may start to oscillate after turning off the noise. Phenomenologically, core nets can be divided into distinguishable states with respect to the dynamical states of population. 1) Core networks with change in the number of machines but not of tapes. 2) Core networks without change in the number of machines or of tapes. 3) Core networks with change in both numbers. Note that a core network without any change in the number of machines and tapes may have temporal oscillation in population amplitude. Emergence of such temporal oscillation depends on the sustained network topology. 
A minimal self-replicating loop( Fig.1-a)) is also an example of core net. It cannot sustain an active error by definition. All the examples of complex core networks which appear at mid external noise $(0.05 \leq \epsilon<0.1)$ have high active error rates. By increasing the amount of external noise more, an attainable core net again loses complexity, becoming a minimal self-replicating loop.

In Fig.4, we depict an active error rate of core nets as a function of external noise, which are attained by turning off external noise at time step 2000.
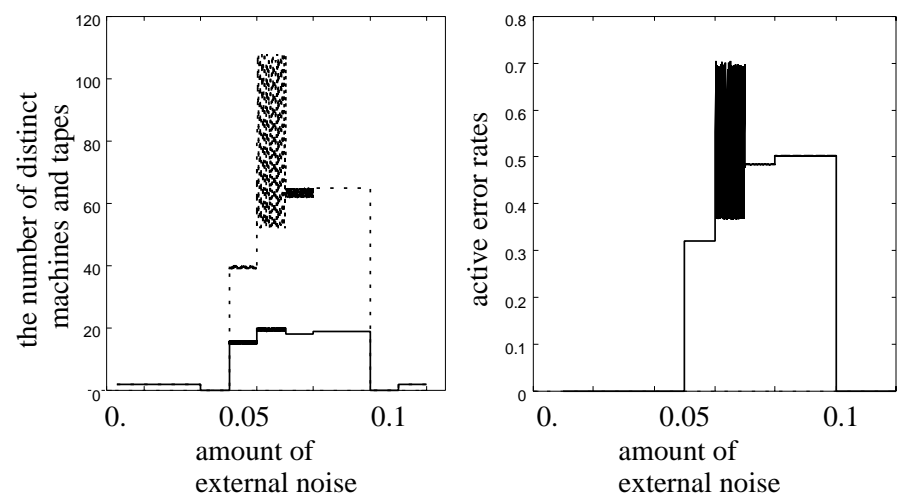

Fig. 4. Temporal evolution of the distinct numbers of machines and tapes(left figure) and those of active error rates( right figure) during time step [2500, 3000] are depicted as a function of external noise. The parameters of population dynamics are $c_{m / t}=d_{m / t}=0.6$.

This diagram depends on the initial configuration of machines and tapes. Some initial configurations never attain any core networks. However the general tendency is that complex core networks emerge in he mid range of $(0.05 \leq \epsilon<$ 0.1 ) of external noise. Namely, there exist upper and lower bound on external noise to evolve rich core networks. Core networks with a fixed point state are more attainable than ones with oscillating states. A core network at external noise of 0.05 contains a rather smaller number of machines than the other core networks. It has been found that this network uses more 0-rich tapes, which contain a bit of state 0 more than that of 1 , in autocatalytic loops. Whereas the other core networks in a fixed point state use more 1-rich tapes, which contain a bit of state 1 more.

The other important criterion to distinguish network states is to investigate the embedded autocatalytic loops. Since Eigen and Schuster's pioneering work [6], notion of autocatalyticy has been known as a useful razor. We use this notion to dissect core networks in the next section. 


\section{$5 \quad$ Embedded autocatalytic loops}

Machines to be sustained in a system should possess their description tapes. Evolution from a simple self-replicating loop to complex ones are depicted in Fig.5, where we express a system by actual machine-tape reaction graphs.

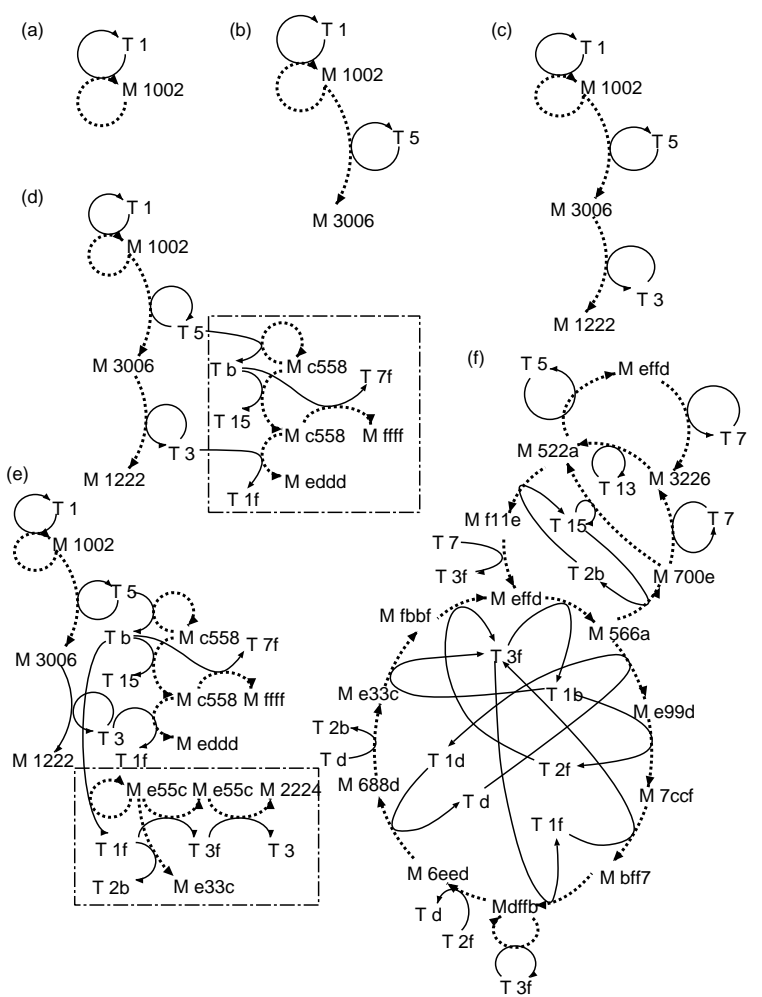

Fig. 5. Evolution from a simple self-replicating loop to a complex network a) An initially existing a minimal self-replicating loop, where a machine $M_{1002}$ replicates itself by reading the tape $T_{1}$. A net a) will be successively exploited by $M_{3006}$ with $T_{5}$ (b)then by $M_{1222}$ with $T_{3}$ (c). The net which is depicted on d) is a net c) with a parasitic network(shown in a box) hanging on it. This is named a parasitic net, since as a network it depends on a net c) for the input tapes $T_{5}$ and $T_{3}$ to maintain its structure. In a network e), a hyper-parasite(shown in a box) hanging on a parasite net in d) is depicted. Embedded autocatalytic loops which are found in the core network after turning off external noise are depicted in f). We can see four independent double autocatalytic loops here. Two are of the Eigen-Schuster type and two are of the double loop types.

In Fig.5-a), a machine $M_{1002}$ copies itself by reading its description tape $T_{1}$. This minimal self-replicating loop exemplifies Eigen-Schuster's autocatalytic net- 
work type. Namely, a tape is self-replicated without error. In high noise regime, the minimal loop is gradually destabilized by side chains. Fig.5 c) and d) show a successive appearance of parasitic networks. A structure obtained by subtracting a net c) from a net d) gives a parasite network. This parasite network depends two input tapes $\left(T_{3}\right.$ and $\left.T_{5}\right)$ on a network c). Similarly, a structure obtained by e) minus d) gives a hyper parasite, which depends a tape $T_{b}$ on the first parasite network. Without a host network c), successive parasite networks will be extinguished.

A finally established core network is a combination of several loops which are autocatalytic. With respect to machine sets only, a closed loop is defined as a chain of $k$ machines where a machine $M_{j}$ generates a machine $M_{j+1}$, the final machine $M_{k}$ generating $M_{1}$. If a tape set needed to sustain the machine loop is self-reproduced by the machine loop, we say that a loop satisfies an autocatalytic condition.

As special case, the autocatalytic condition is locally satisfied; each tape is self-replicated. A network of this type is called the Eigen-Schuster type. Since each tape self-replicates, no active error exists in this network type. However, such autocatalytic condition is not locally satisfied in general. In the noisy environment a more general network which globally satisfies the condition will be expected. Difference between two possible network types are depicted in Fig.6.

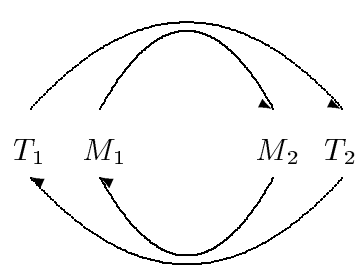

a)

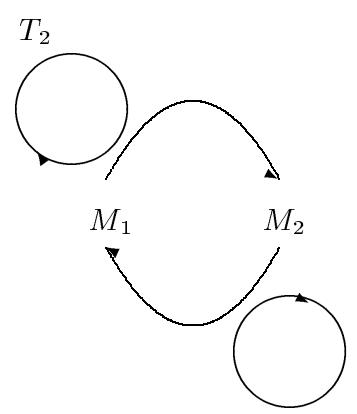

b)
$T_{1}$

Fig. 6. Illustration of two different autocatalytic loops. They are Eigen-Schuster type( the right figure) and the double loop type(the left figure). Tapes are replicated without errors in Eigen-Schuster type. In the double type, both machines and tapes are replicated into the other.

In the general case of Fig.6-b), not only machines but also tapes form a loop structure. Hence we call the expected autocatalytic networks emerging in high noise regime a double autocatalytic network. In the low noise regime, a network can sustain its structure by Eigen-Schuster type. But for the high noise regime, 
it is difficult to maintain prefect replication. It then switches to the double loop structure. Since Eigen-Schuster type only allows replication without error, we call this type a DNA-like replicating system. On the other hand, a double network type edits tapes, which we call a RNA-like transcribing system.

Compared with core nets in a fixed point state, we find that cores in oscillatory states change the number of double loops in time. Details will be discussed elsewhere [7].

\section{6 discussions}

We have studied the coevolution of machines and their description tapes. We note that a minimal self-replicating loop, composed of one machine and one tape, emerges under influence of external noise.

When external noise is elevated, a minimal self-replicating loop is exploited by parasitic networks. Population of machines and tapes then begins to show unstable oscillations. In the mid regime of external noise, a system will evolve into a stable network against external noise spontaneously. When a network attains this state, it is stably sustained even after external noise is removed. We therefore call the network acquiring such noise stability a "core network". The core network consists of many autocatalytic loops, being a fixed point or oscillatory state.

A minimal self-replicating loop has no replication errors, and corresponding to the simplest Eigen-Schuster's autocatalytic network. Such autocatalytic network is only stable below some noise level. On the other hand, a high active error rate is sustained in core networks in the high noise regime. Self-replication with errors replaces those without active errors in the noisy environment.

This autocatalytic network with high active error rates consists of double loops of machines and tapes. Namely, each machine replicates a successive machine on a loop. On the other hand, description tapes of the machines composing of a loop are replicated as a whole, not individual tapes.

A transition from an unstable state to a core network state is similar to what we have seen in a previous host parasite model [8]. In the model, chaotic instability is shared by almost all species by sustaining a high mutation rate, leading to weak high-dimensional chaos, termed "homeochaos". In this paper, a role of host and parasite emerges spontaneously in a network. Interaction of host and parasite loops causes dynamic instability as well. The core structure suppresses the instability and as the result a high mutation rate is sustained in the present model.

The idea of introducing two different errors provides a new interpretation of self-replication. A network absorbs external noise as an active error of machine function. Namely, passive error caused by external noise is replaced by a deterministic error.

In real biological systems, DNA is replicated individually without active errors. RNA is also a mere copy of DNA. However, we have an example [9] which may be related to our double autocatalytic network. The authors showed that 
DNA of macronucleus is generated from DNA of micronuclear in Oxytricha nova. DNA of micronuclear is transcribed once into RNA. Then the exons are completely rearranged and reverse transcribed into the DNA of macronucleus. If editing and reverse transcribing RNA is more stable than replicating DNA itself, an active error as editing will be favored in some genetic systems.

In order to enhance a desirable mutation, we have to generate mutations not by chance but by a deterministic process. In order to suppress the undesirable mutation, we have to change our coding structure, for example by replacing unstable description tape with more stable tapes. However it is difficult to chose stable coding generally. An optimal coding of a machine may lead to the worst coding of the other. This causes a temporal oscillation even in a core network. Note that self-organization of an autocatalytic network implies self-organization of a coding system [10]. How to improve coding cannot be determined locally but should be done in a network context. Alternation of coding driven by external noise has also been reported recently [11]. In their systems, a multi coding system is developed in the high noise regime.

\section{Acknowledgment}

One of the authors(T.I.) would like to thank Pauline Hogeweg for stimulating and critical discussions on this work, and her bioinformatic group for their heartful courtesy while he was staying in Utrecht. His stay was supported by NWO (Netherlands Scientic Research Foundation). This work is partially supported by Grant-in-Aids (No.06854014) for Scientific Research from the Japanese Ministry of Education, Science and Culture.

\section{References}

1. J.von Neumann, Theory of Self-reproduction(1968)

2. This is a main theme discussed by D.Hofstadter in his book, Gödel, Escher, Bach: An eternal Golden Braid(Penguin Books, 1980).

3. M. Eigen, Sci. Am. (1993) July, 32. Also see P.Schuster, Physica 22D(1986) 100.

4. See also, M.Andrade et al. Physica D 63 (1993) 21.

5. See e.g., D.J.Farmer, S.S. Kauffman, N.H.Packard and A.S.Perelson, Ann.Rev. NY Acad.Sci. 504(1987)118.

6. M.Eigen and P.Schuster, Hypercycle(Springer-Verlag, 1979).

7. T.Ikegami and T.Hashimoto, to be submitted.

8. See e.g., K.Kaneko and T.Ikegami, Physica D 56 (1992) 406:; T.Ikegami and K.Kaneko, CHAOS 2 (1992) 397.

9. A.F.Greslin, Proc.Natl.Acad.Sci. USA 86(1989)6264.

10. P.R.Wills, J.Theor.Biol. 162(1993)267.

11. P.Hogeweg and B.Hesper, Computers Chem. 16 (1992) 171. 